\title{
Series cartográficas y base de datos de información geográfica producidas en España
}

Javier González Matesanz, Javier García García, Juan Luis Bermúdez González, Adolfo Dalda Mourón, y Francisco Maza Vázquez

\section{Resumen:}

Las tareas de elaboración o actualización de la cartografía nacional de un país deben iniciarse con el análisis pormenorizado de las opciones disponibles, y aprovechar la experiencia y conocimientos adquiridos por otros productores en la elaboración de sus mapas, tanto en técnica como en estética. En el Instituto Geográfico Nacional se dispone de una amplia variedad de productos cartográficos básicos y derivados cubriendo una gran variedad de escalas y productos que van desde 1:25.000 hasta escalas muy pequeñas como 1:8.000.000. En algunas series se dispone de una dualidad entre la base de datos de Información Geográfica y la versión mapa o cartográfica de la misma. De esta forma, se conserva una resolución aproximadamente mitad en la base de datos, es decir BTN25 (Base Topográfica Nacional 1:25.000) tiene una resolución 1:10.000 (aproximadamente) como fuente principal de MTN25 (Mapa Topográfico Nacional), y BTN100 (Base Topográfica Nacional 1:100.000) con una resolución 1:100.000 que es el soporte fundamental del Mapa Provincial 1:200.000. Sin embargo, debido a los ciclos de vida de escalas pequeñas, por ejemplo 1:1.000.000, es necesario realizar en ellas actuaciones más frecuentes que en escalas grandes que deben ser aprovechadas en la medida de lo posible en estas escalas, aunque sea generalmente solo a nivel semántico.

Palabras clave: Series cartográficas/MTN25/MTN50/BTN25/Cartografía oficial

\section{Abstract:}

Making or updating the National cartography of a country requires a well "in deep" analysis of any option available and to take advantage and knowledge of other cartography producers, in the technique and esthetic sense. In the Instituto Geográfico Nacional of Spain we do have a great range of basic and derived 
cartography from 1:25.000 scale to lesser ones as 1:8.000.000. In some series we have a duality between the Geographic Information Database and the map version, or the cartographic version. In this sense, we retain half the resolution in the database, this means that BTN25 (National Topographic Database 1:25.000) have 1:10.000 resolution (approximately) and BTN100 (National Topographic database 1:100.000) is the geometrical support of the Province Maps 1:200.000. However, due to the lifecycle of smaller scales, for instance 1:1.000.000, a shorter updating period is needed than in greater ones that should be taken into account, as much as can, although this can be done only in the semantic part of the geographical object.

Keywords: Cartographic Series/MTN25/MTN50/BTN25/Official cartography

González Matesanz, Javier (fjgmatesanz@fomento.es), Instituto Geográfico Nacional de España/Universidad de Alcalá; García García, Javier (fjggarcia@fomento.es), Instituto Geográfico Nacional de España; Bermúdez González, Juan Luis (jlbermudez@beritec.es), BERITEC Cartografía y catastro SL; Dalda Mourón, Adolfo (adolfo.daldamouron@gmail.com), Instituto Geográfico Nacional/Universidad de Alcalá; Maza Vázquez, Francisco,(francisco. maza@uah.es), Universidad de Alcalá. 


\section{ANTECEDENTES}

El Instituto Geográfico Nacional fue creado en 1870 siendo su primer Director el Coronel Ibáñez e Ibáñez de Ibero. Sus misiones consistían en realizar los trabajos relativos a la determinación de la forma y dimensiones de la Tierra, triangulaciones geodésicas de diversos órdenes, nivelaciones de precisión, triangulación topográfica, topografía del mapa y de catastro, así como las cuestiones relativas a pesos y medidas. Hoy en día, tras 143 años de tradición cartográfica, los rápidos canales de difusión de la Información Geográfica (IG) obligan a replantear métodos mucho más eficientes y automáticos, en la medida de lo posible, para satisfacer las demandas de los usuarios, tanto corporativos como privados.

Las Bases y las Series Cartográficas Nacionales del Instituto Geográfico Nacional (IGN) tienen por objetivo producir y actualizar la información geográfica y cartográfica institucional que compete al IGN y promover la disponibilidad de información geográfica en cooperación con las Administraciones Públicas, satisfaciendo sus requerimientos para la prestación de servicios y productos a la sociedad (García-Asensio, 2009) y, pueden dividirse en dos grandes bloques, los destinados a Sistemas de Información Geográfica (SIG) y la cartografía digital e impresa. Los primeros comprenden la Base Topográfica Nacional 1:25.000 - BTN25 que constituye el soporte geométrico y semántico fundamental para la Cartografía Básica del Estado y la Base Cartográfica Numérica 1:200.000-BCN200. El segundo de los bloques lo componen: el Mapa Topográfico Nacional 1:25.000 - MTN25 (Cartografía Básica del Estado), el Mapa Topográfico Nacional 1:50.000 - MTN50, el Mapa Provincial 1:200.000, los Mapas Autonómicos, el Mapa de España 1:500.000, los Mapas Generales de España 1:1.000.000 y 1:2.000.000 y la Cartografía en Relieve. 
Figura 1. Hojas del Mapa Topográfico Nacional 1:50.000 de ediciones separadas más de 100 años

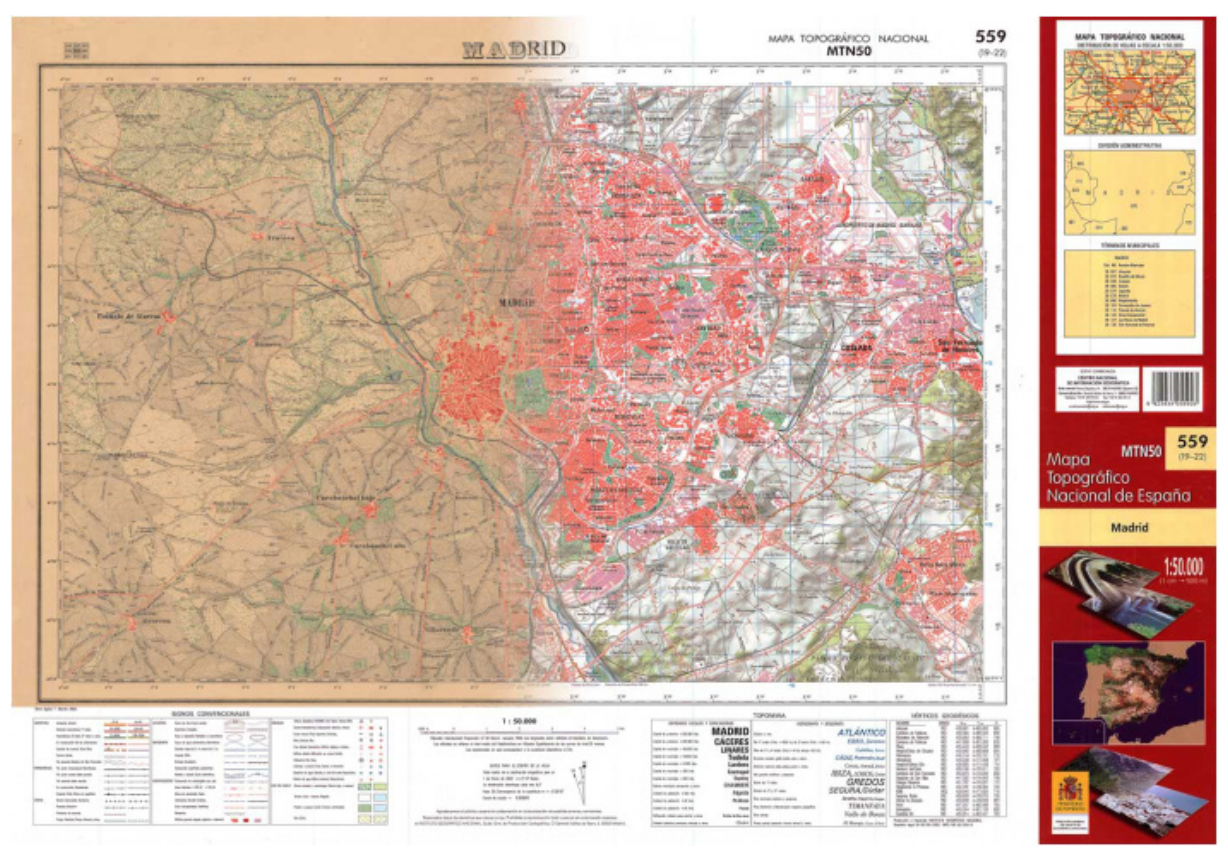

Por otra parte, en los últimos años se ha realizado un enorme esfuerzo normativo en materia de IG disponiendo en la actualidad de un vasto número de normas que permiten articular y ordenar no solo la producción cooperativa e interoperable de IG sino también acercar y poner a disposición de los usuarios estos recursos, un listado de disposiciones legales existentes que afectan a la IG producida por la administración sería el siguiente (Lázaro y Jiménez de Cisneros, 2010):

- Ley 11/1975, de 12 de marzo sobre Señales Geodésicas y Geofísicas.

- Ley $7 / 1985$, de 2 de abril, Reguladora de las Bases del Régimen Local

- Ley 7/1986, de 24 de enero, de Ordenación de la Cartografía.

- Real Decreto Legislativo 1/2004, de 5 de marzo, por el que se aprueba el texto refundido de la Ley del Catastro Inmobiliario.

- Ley 27/2006, de 18 de julio, por la que se regulan los derechos de acceso a la información, de participación pública y de acceso a la justicia en materia de medio ambiente (incorpora las Directivas 2003/4/CE y 2003/35/CE).

- Ley 11/2007, de 22 de junio, de Acceso Electrónico de los Ciudadanos a los 
Servicios Públicos. Ley 37/2007, de 16 de noviembre, sobre Reutilización de la Información del Sector Público.

- Real Decreto 2421/1978, de 2 de junio, por el que se aprueba el Reglamento de la Ley

- 11/1975, de 12 de marzo, sobre Señales geodésicas y geofísicas.

- Real Decreto 417/2006, de 7 de abril, por el que se desarrolla el texto refundido de la Ley del Catastro Inmobiliario, aprobado por el Real Decreto Legislativo $1 / 2004$, de 5 de marzo.

- $\quad 11 / 1975$, de 12 de marzo, sobre Señales geodésicas y geofísicas.

- Real Decreto 417/2006, de 7 de abril, por el que se desarrolla el texto refundido de la Ley del Catastro Inmobiliario, aprobado por el Real Decreto Legislativo $1 / 2004$, de 5 de marzo.

- Directiva 2007/2/CE del Parlamento Europeo y del Consejo de 14 de marzo de 2007 por la que se establece una infraestructura de información espacial en la Comunidad Europea (INSPIRE).

- Real Decreto 663/2007, de 25 de mayo, por el que se aprueba el Estatuto del Centro Nacional de Información Geográfica.

- Real Decreto 1071/2007, de 27 de julio, por el que se regula el Sistema Geodésico de Referencia Oficial en España.

- Real Decreto 1545/2007, de 23 de noviembre, por el que se regula el Sistema Cartográfico Nacional.

- Real Decreto 4/2010, de 8 de enero, por el que se regula el Esquema Nacional de Interoperabilidad en el ámbito de la Administración Electrónica.

- Real Decreto 638/2010, de 14 de mayo, por el que se modifica y desarrolla la estructura orgánica básica del Ministerio de Fomento.

- Orden FOM/956/2008, de 31 de marzo, por la que se aprueba la política de difusión de la información geográfica generada por la Dirección General del Instituto Geográfico Nacional.

- LISIGE. Ley sobre las Infraestructuras y los Servicios de Información Geográfica, aprobada el jueves 17 de junio de 2010.

Consecuentemente, el nuevo "teatro de operaciones" en cuanto a la producción de IG se refiere, cristaliza el carácter de actividad instrumental de la cartografía y permite la reutilización e interoperabilidad de la IG generada por distintos actores y niveles de administración a través de la LISIGE Esta Ley impulsa el aprovechamiento de las nuevas tecnologías en los servicios de información geográfica, 
potenciando su calidad técnica, y teniendo en cuenta su utilidad como servicio público.

La LISIGE dota de rango legal el Sistema Cartográfico Nacional (RD 1545/2007), hace efectiva la directiva INSPIRE, y marca como mecanismo prioritario la cooperación entre las Administraciones y la política de difusión libre de la Información Geográfica digital generada. Regula las competencias en relación con los servicios de cartografía oficial y la Infraestructura de Información Geográfica de España, cuya constitución y mantenimiento corresponde al Consejo Superior Geográfico. EI IGN por su parte actúa como coordinador y operador de esta infraestructura. En su Disposición adicional segunda impulsa una política de difusión libre de la información geográfica de referencia y de la cartografía topográfica digital garantizando a su vez el uso de las lenguas oficiales del Estado. Materializa así mismo la Información Geográfica de Referencia constituida por: El Sistema de Referencia Geodésico, El Sistema Oficial de Coordenadas, La toponimia del Nomenclátor, Geográfico Básico de España y otros Nomenclátores, Delimitaciones Territoriales del Registro Central de Cartografía, El Inventario Nacional de Referencias Geográficas Municipales, La parcela catastral (DG Catastro + Diputaciones Forales), la Altimetría y batimetría, Redes de transporte, Hidrografía (Ley de Aguas), Lugares protegidos Internacionales a autonómicos, Ocupación del suelo, Geología y geomorfología, Direcciones y Entidades de población. Constituyendo 14 temas a los que se añaden 21 temas pertenecientes a los datos temáticos fundamentales del Anexo II y 8 temas de datos temáticos generales.

La Directiva INSPIRE establece una infraestructura de "información de referencia", es decir, de una cobertura completa, actualizada y armonizada de Europa, que se realiza de manera ésta información sea recogida una vez y mantenida donde se logre máxima efectividad, la interoperabilidad hace posible combinar la información geográfica con total continuidad para toda Europa desde fuentes diversas y compartirla entre usuarios y aplicaciones, lo que provoca una coordinación a cuatro niveles de Administración. 
Figura 2. Algunas representaciones de diversos productos, de la esquina inferior izquierda y siguiendo un orden horario: Ortofoto 25cm de resolución, BTN25, MTN25, MTN50, 1:500.000, MP200, BTN100, imagen SPOT5

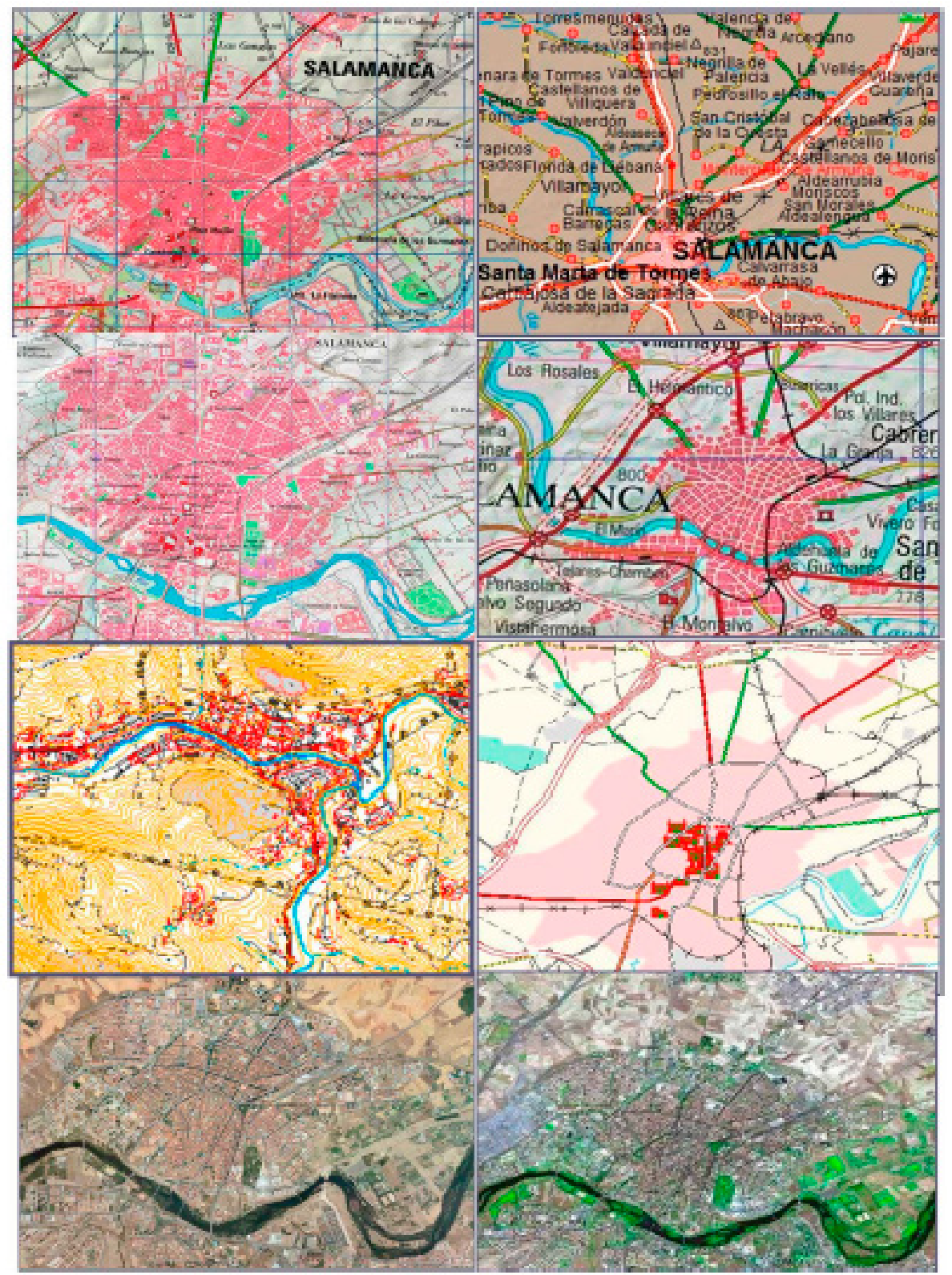




\section{BCN25 y BTN25}

Armonización implica no solo el cumplimiento de la LISIGE sino, a efectos prácticos, el desarrollo de un catálogo de fenómenos geográficos a una escala común entre Comunidades Autónomas (CCAA). La Comisión Especializada de Normas Geográficas fue la encargada de realizar este trabajo, generando la Base Topográfica Armonizada cuya versión 1.0 fue aprobada por la por la Comisión Técnica Permanente del Consejo Superior Geográfico en su reunión del 4 de noviembre de 2008. Uno de los objetivos de BTA es la interoperabilidad de la información geográfica digital de la cartografía topográfica 1:5.000 y 1:10.000 generada por la Administración, tanto a nivel nacional como regional y local. Este hecho obliga en cierta forma a desarrollar un protocolo de case entre los fenómenos geográficos al llegar a la frontera entre dos CCAA de forma que el producto final sea una base de datos de información geoespacial continua.(CENG, 2007). Por consiguiente, es preciso realizar convenios con las CCAA de manera que la armonización entre la cartografía producida por las Agencias Cartográficas Regionales (AGR) de las CCAA y la propia generada por el IGN permitan dar la misma visión del territorio a un usuario. Estos convenios se sustentan en la generación de un producto común de aprovechamiento mutuo que "engrana" las dos producciones cartográficas y permite producir la Base Topográfica Nacional 1:25.000 (BTN25) de forma cooperativa.

A mediados de 2005 la Subdirección General de Geodesia Cartografía inició los trabajos de formación de la BTN25 y abandonando el producto precedente BCN25 (Base Cartográfica Numérica 1:25.000) cuya geometría procedía directamente del MTN25 (Mapa Topográfico Nacional 1:25.000) y que por consiguiente adolecía de los desplazamientos y restricciones necesarios en los procesos de redacción cartográfica de esta serie.

BTN25 por el contrario tiene una geometría que procede de restitución, con una calidad posicional próxima a una escala 1:12.500, en 3D y con todo el contenido semántico de MTN25. Para realizar esta transición se ideó un proceso que permitiera rescatar la geometría de restitución por una parte y la riqueza semántica generada en BCN25 mediante una correlación entre ficheros realizada de forma semiautomática. 

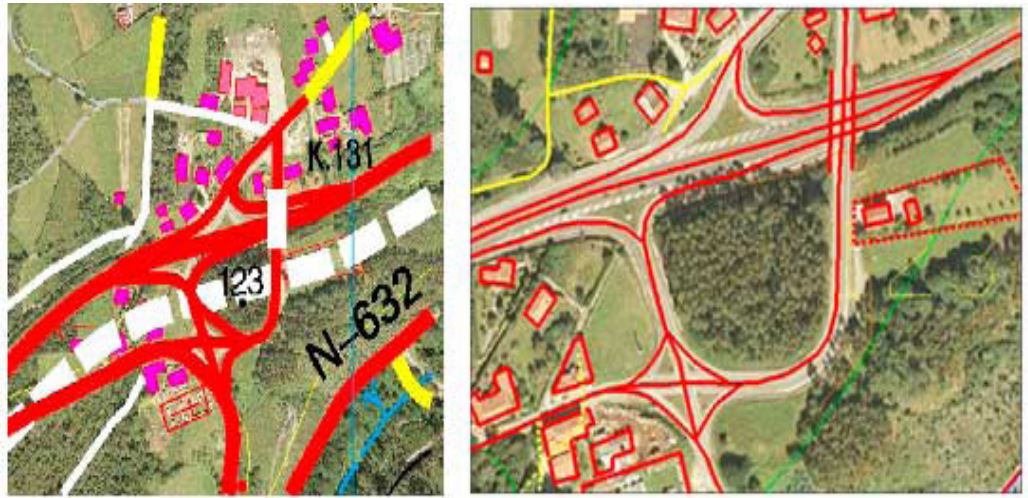

Figura3. BCN25 (Izda) y BTN25 (Dcha.)

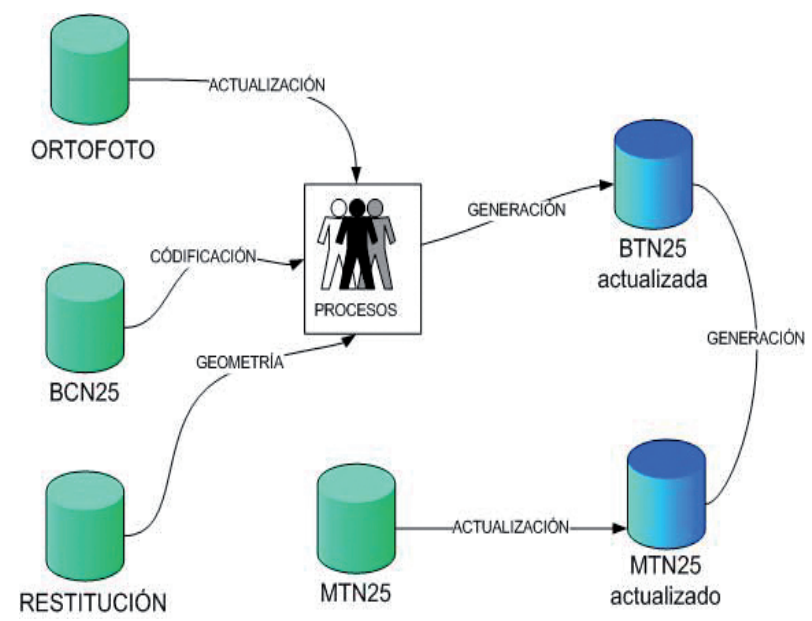

Figura 4. Procedimiento de recuperación geométrica.

De esta manera se consigue, por una parte generar de una forma relativamente rápida una versión primera de BTN25 para su actualización rescatando la geometría de restitución y todo el trabajo de enriquecimiento semántico de BCN25. A partir de esta BTN25 se genera una versión de MTN25 con la que se actualiza este último.

Una de las tareas que más tiempo consumen es llevar a cabo los controles de calidad tanto en BTN25 como en BTA+, con el espíritu de acelerar y automatizar cuantos controles sean necesarios, se ha diseñado un procedimiento que permite, 
tanto en la fase de captura o edición, como en una revisión posterior aplicar reglas semánticas. Las reglas semánticas consisten en la formalización del conocimiento de los controles de calidad en forma de consultas espaciales tanto para captura, formación y edición. Las Reglas Semánticas son las normas de captura y formación; las de edición son las normas de edición escritas y no escritas, es decir, la formalización del conocimiento de los distintos revisores. Se han generado reglas tanto para BTN25 como para MTN25 para su implementación en tiempo real (captura y formación) y en post-proceso (validación de captura y formación). Las de Edición se implementan en la fase de validación de hojas ya creadas, es decir, en Edición se trata de pasar controles automáticos a las hojas contratadas o producidas por medios propios, con el objetivo de rebajar tiempo de revisión, en principio se trata de implementarlas desde la captura, evitando no sólo la trasmisión de errores, sino evitando algo más importante que es no llegar a cometer el error, tenerlo controlado en la fase de captura y actualización. Es posible formalizar este conocimiento de muy diversas maneras, y siempre se plantea de forma que exista el mejor equilibrio posible entre "el estado actual de BTN25" y "lo que se desea tener" de forma que no se incluyan excesivas reglas que no se cumplan en las propias especificaciones actuales o sea muy laboriosa su introducción, un ejemplo de reglas semánticas son:

- Entidad de población debe contener dos o más Edificaciones

- Punto de cruce de Río (Permanente) con Vías de comunicación, debe tocar Paso elevado lineal (sin pasarela) o Paso elevado superficial

- Embalse debe unirse a Presa y Río

- Unión de Presa debe unirse a Embalse

- Punto de cruce de Autopista/Autovía con otra vía debe tocar Paso elevado lineal (sin pasarela), superficial, o Túnel Lineal

- Frontera de Isla debe coincidir exactamente con Curva de nivel cota 0

- Punto intermedio de Embalse no debe estar contenido en Presa o Río

- Extremo de Línea eléctrica debe terminar en Torre de alta tensión, Transformación eléctrica o Línea eléctrica

- Edificación puede ser solapado por Punto acotado, Antena, Vértice geodésico, Límite administrativo, Curva de nivel, Línea eléctrica o Edificación

- Línea de costa debe coincidir con Curva de nivel de cota 0 


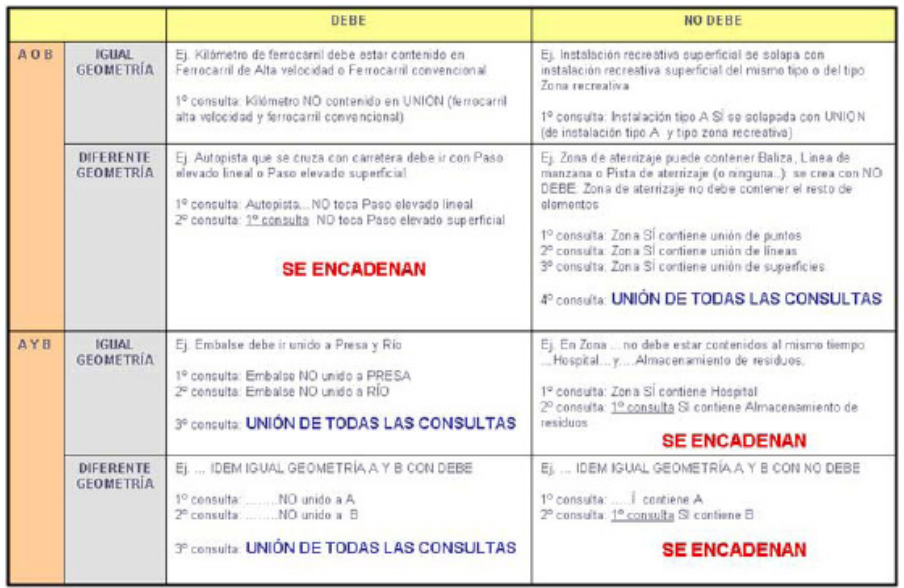

Figura 5. Ejemplo de aplicación de reglas semánticas
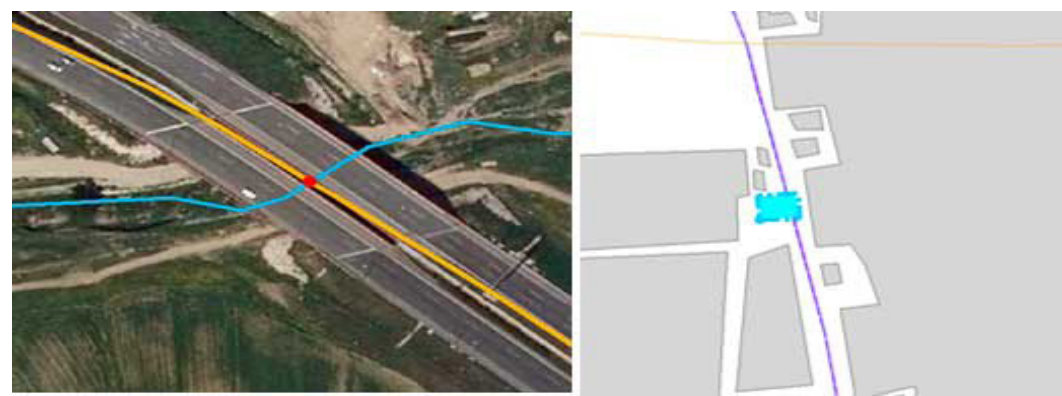

Figura 6. Ejemplo de aplicación de reglas semánticas

En la Figura) se puede observar un ejemplo de regla semántica donde se ha detectado automáticamente que un río se cruza con una autovía y no existe un paso elevado. Y en la misma figura a la derecha se puede apreciar la detección de elementos superpuestos, en este caso, un edificio superpuesto a una vía de comunicación.

\section{ACTUALIZACIÓN DE MTN25}

Una vez terminada la primera versión completa de MTN25 hace ya algunos años, se vienen haciendo sucesivas actualizaciones, bien partiendo de BTN25 producida de forma armonizada con las CCAA, bien por medios propios. Evidentemente, MTN25 es un producto cartográfico puro y en consecuencia sufre de desplazamientos, simbolizaciones y selección de elementos propios de los 
procesos habituales de redacción cartográfica. Por si fuera poco, para la actualización de MTN25 se utiliza como referencia BTN25 pero no de forma exclusiva, sino que BTN25 se ha convertido en una fuente más para su actualización y como hemos citado anteriormente existe una diferencia de escala, mientras BTN25 tiene tecnología SIG y una geometría próxima a la escala 1:10.000, MTN25 es un producto puramente cartográfico a escala 1:25.000. Para agilizar la actualización se ha desarrollado un procedimiento de detección de cambios entre BTN25 y MTN25, de esta manera se actualiza solo aquellos elementos que han cambiado, añadido o borrado. Algunos de los problemas habituales en esta fase son: no todos los elementos BTN son representados en el mapa, varios elementos BTN pueden ser representados por un elemento Mapa, un elemento BTN puede ser representado por dos o mas elementos mapa, la posición de un elemento BTN y su representación mapa pueden estar desplazados, la geometría BTN y mapa no son la misma, en mapa se representa como símbolos elementos superficiales.

En la figura anterior se pueden observar cambios detectados en edificaciones, estas o bien son nuevas o bien son edificios que han modificado su superficie o ésta difiere significativamente en MTN25. El propio proceso de detección de cambios transmite los mismos al equipo de MTN25 ya generalizados de forma que con un proceso semi-automático secuencial se van recorriendo uno a uno aceptándose o rechazándose.

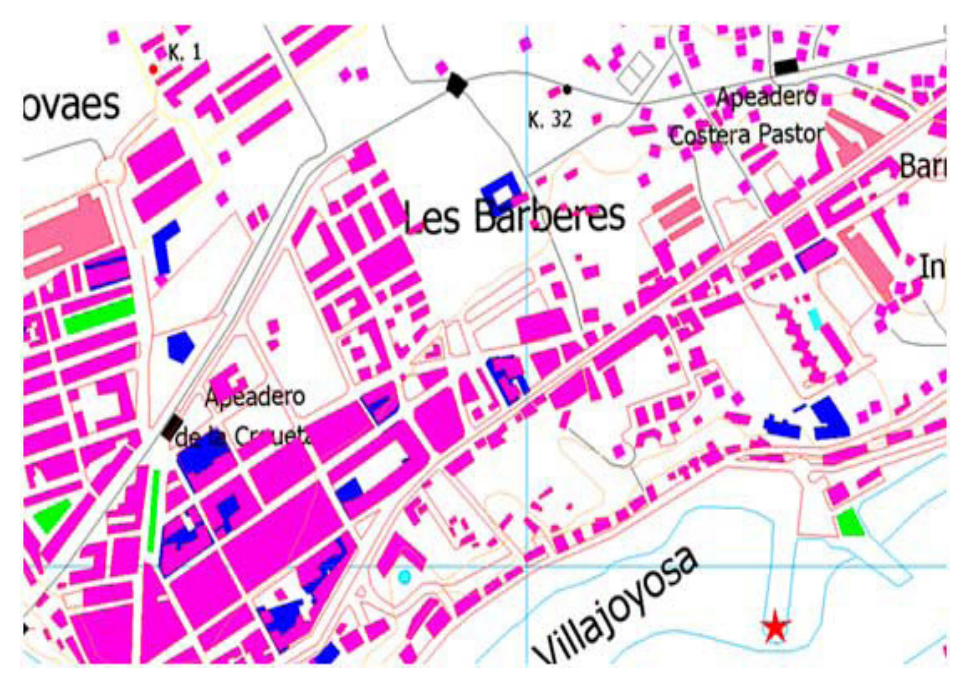

Figura 7. Ejemplo de detección de cambios. 


\section{PEQUEÑAS ESCALAS}

Si todos los procesos citados anteriormente son complejos, el nivel de complejidad crece exponencialmente según aumenta el denominador de la escala, esto es, un mapa a escala 1:200.000 sufre de muchos más procesos de redacción cartográfica que uno a escala 1:25.000 (Merino y González.-Matesanz, 2009), consecuentemente la divergencia entre la versión cartográfica y la base de datos es mayor. Por otra parte, las necesidades de actualización son mucho más críticas cuanto menor es la escala del mapa, si para MTN25 se tiene un objetivo de 5 años, en el caso de MP200 (Mapa Provincial 1:200.000) el objetivo es de uno o dos años y consecuentemente debe tener una actualización independiente de MTN25. Si en escalas grandes se pueden encontrar sinergias con las CCAA, en escalas pequeñas el CEGET (CEntro Geográfico del Ejército de Tierra) no solo tiene una problemática similar sino también una tecnología análoga. Es obvio que es posible establecer una colaboración en la producción de bases topográficas a escalas 1:100.000 y 1:200.000, pues de nuevo la base topográfica (BCN200) que subyace en MP200 tiene una resolución doble, de manera similar a cuando se compilaban las minutas al formar una hoja de forma analógica (González.-Matesanz, 2006).

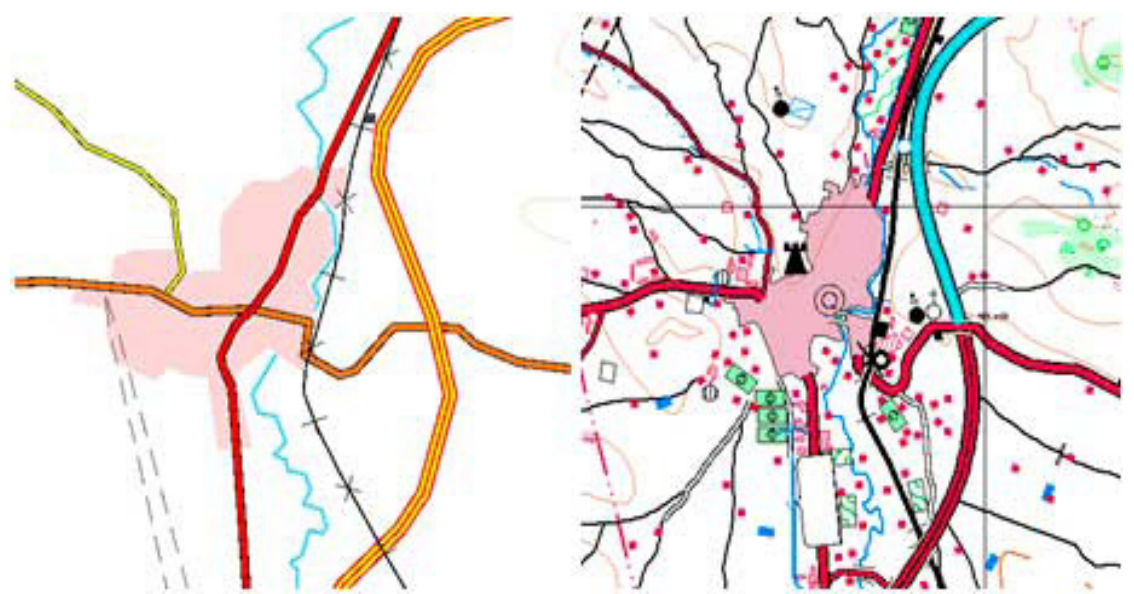

Figura 8. Misma realidad representada por BCN200 y serie C respectivamente.

Como trabajo previo se realiza un análisis de los dos modelos de datos, serie C-CEGET y BCN200-IGN resultando como sigue: 
- Modelo Serie C - CEGET

- Modelo cartográfico

- Finalidad para uso militar

- 205 clases de entidad (149 geometría + 56 textos)

- Modelo BCN200 - IGN

- Modelo SIG

- Finalidad para uso civil

- 47 clases de entidad (geometría)

La manera más sencilla que satisface los requerimientos de ambos organismos pasa por la generación de una base topográfica a escala 1:100.000 compartida que permita la producción impresa de la serie $\mathrm{C}$ y que mediante procesos de generalización (Dávila, 2007) sea capaz de producir una versión 1:200.000 que de lugar por una parte al 250.000 del CEGET y al MP200 del IGN. En una fase posterior se abordará continuar con la generalización de esta base 200.000 a 500.000 para la producción de ME500 (Mapa de España 1:500.000) (Celada y Núñez, 2007) y el 500.000 de CEGET.

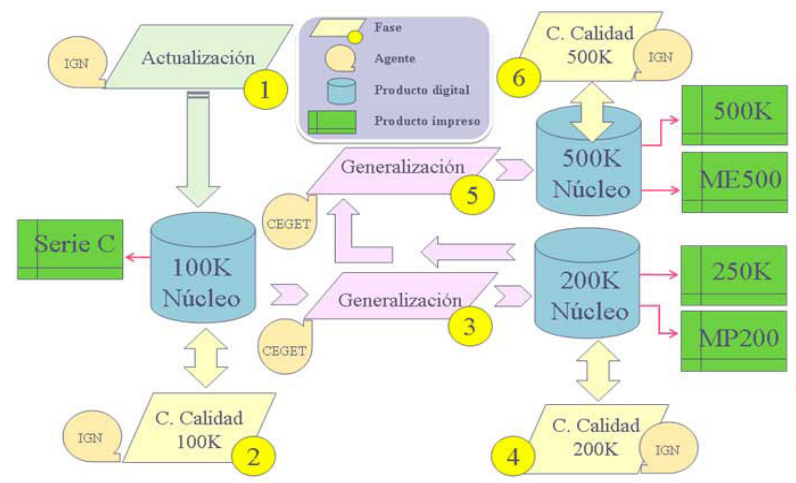

Figura 9. Flujo de trabajo colaborativo con CEGET.

Este modelo inicial a escala 1:100.000 (Base100) se completa de forma conjunta y a mediados de 2010 se inicia la primera hoja piloto de producción compartida.

- 55 clases de entidad 


\section{o 31 aporta IGN}

- Límites administrativos

- Usos de suelo

- Edificaciones y núcleos urbanos (excepto muralla)

- Comunicaciones (excepto puente, faro y aeropuerto p.)

- Conducciones: estación de telecomunicaciones. y depósito de combustible

- Vértices geodésicos

\section{o 24 aporta CEGET}

- Altimetría

- Hidrografía

- Conducciones (excepto estación de telecomunicaciones. y depósito de combustible)

- Otras: puente, muralla, faro, aeropuerto $p$.

\section{FLUJO IDEAL DE TRABAJO}

En la actualidad todas las cadenas de producción se están migrando de tecnología CAD a SIG, esto implica un drástico cambio tanto en tecnología como en forma de actuar. El reto es mantener en determinadas escalas o productos estratégicos al menos dos representaciones de un mismo fenómeno geográfico, la versión "fuente" o topográfica con geometría coincidente a la escala con el mundo real, y la versión "cartográfica" simbolizada a una escala concreta.

Una vez se han consolidado las bases topográficas, BCN200 o BTN25, bien sea por medios colaborativos o producción propia, pasan a una base de datos centralizada Oracle Spatial 10g. A partir de esta base de datos se puede actualizar por medios propios utilizando una simbolización ligera (González.-Matesanz et al., 2007), esto es, los fenómenos geográficos son representados de forma coherente con la realidad terreno a la escala escogida sin sufrir procesos de redacción cartográfica. En caso de necesitar versión mapa se utiliza Geomedia MapPublisher (Merino, 2008) para generar una versión cartográfica del mismo fenómeno, así se puede simbolizar, desplazar, seleccionar y borrar elementos sin perder la coherencia con la versión "fuente" y con resolución de conflictos cartográficos de manera automática y asistida, las sucesivas actualizaciones se realizarán siempre modificando la versión "fuente" y el propio programa se encarga de gestionar únicamente 
las modificaciones. Una vez se obtiene este producto comienza el etiquetado automático, pues en toda base topográfica el nombre del fenómeno o su etiqueta, es un atributo del elemento que puede ser posicionado automáticamente con un éxito aproximado del $80 \%$, el resto es trabajo manual pero que queda almacenado para posteriores ediciones (Celada, 2008). Y finalmente si es necesario se generan sombreados e hipsometría para pasar a plancha de forma directa a través de PDF.

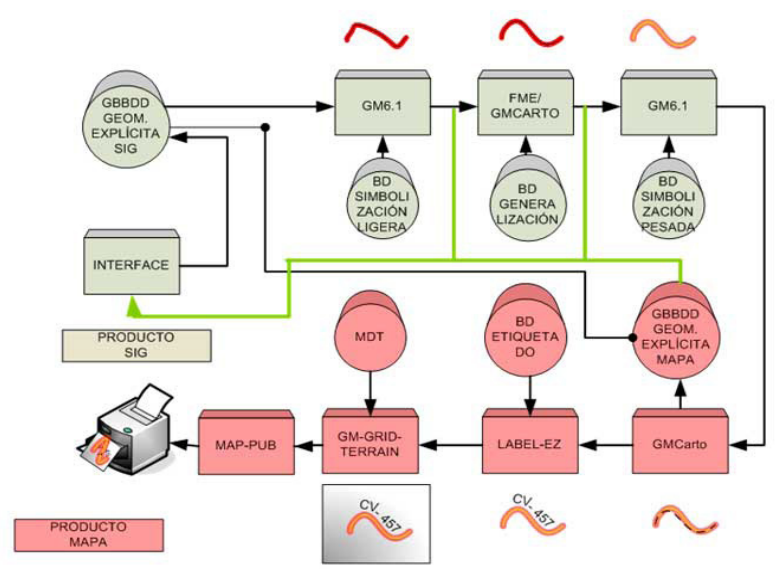

Figura 10. Flujo de producción ideal.

\section{CONCLUSIONES}

Tanto las herramientas normativas disponibles en la actualidad como la propia tecnología permiten la producción cooperativa de información geográfica a distintas escalas y con diversos objetivos. Sin embargo gran parte de los procesos tienen una solución "difusa o borrosa", por ejemplo los de generalización, abocando al productor a soluciones de compromiso que permitan no penalizar en demasía las cadenas de producción. En todo caso, gran parte de los controles de calidad pueden llevarse a cabo de forma automática o semi-automática, y en el peor de los casos asistida, una vez que se migra la tecnología hacia Sistemas de Información Geográfica y se puede formalizar el conocimiento en forma de consultas espaciales tanto en tiempo real como en postproceso. Indudablemente, el usuario actual demanda información geográfica de fácil acceso y actualizada, especialmente en aquellos fenómenos geográficos especialmente sensibles a las personas, redes de transporte y poblaciones, algo que ningún organismo es capaz de producir a la ve- 
locidad deseada de forma independiente y por tanto se ha de establecer un marco de colaboración para la producción cooperativa para lo cual las herramientas normativas existentes son especialmente útiles (por ejemplo el Sistema Cartográfico Nacional) que no solo regula y ordena la producción cartográfica sino que permite el diseño de un verdadero Plan Cartográfico Nacional que satisfaga a productores y usuarios.

\section{AGRADECIMIENTOS}

Este artículo es fruto del trabajo del Grupo de Apoyo y Desarrollo del Área de Cartografía Básica y Derivada: Valeriano Martín-Carrascal, Alfonso Marín, Francisco Sánchez, Francisco Dávila, José Antonio Merino, Tania Gullón, Ana de las Cuevas, Ana Maldonado, Jesús Celada , Eduardo Núñez y Ángela Ruiz, para todos ellos mi más sincero agradecimiento. Es merecido también el agradecimiento a nuestros colegas del CEGET por su dedicación y entusiasmo en el proyecto de colaboración a escalas 100.000 y 200.000 .

\section{BIBLIOGRAFÍA}

- Celada, J., 2008. Pilot Project ME500 and MP200 Prototype. New Cartographic Hardcopy Production Process with GeoMedia Professional Intergraph Annual User Meeting, Las Vegas, Nevada.

- Celada, J. y Núñez, E., 2007. GIS tools implementation in IGN Cartographic Production: ME500 and 'Cartolmage', EuroCartoSIG2007, Zagreb, Croacia. CENG, 2007. Especificaciones BTA, Comisión de Normas Geográficas

- Dávila, F.J., 2007. Generalización en el Instituto Geográfico Nacional de España. In: (publicación interna) (Editor), ICC2007, Moscú.

- García-Asensio, L., 2009. Producción cartográfica en el Instituto Geográfico Nacional, Curso Básico IGN.

- González.-Matesanz, J., 2006. SIG25 status at Cartography Area in IGNE. Internal report, IGNE annual meeting, Valencia.

- González.-Matesanz, J., Celada, J., Martín-Asín, G. y García-Asensio, L., 2007. 
Towards an integral GIS cartographic production in the National Geographic Institute of Spain, Expogeomatica 2007, La Habana, Cuba.

- Lázaro, J. y Jiménez de Cisneros, M.A., 2010. Reparto de competencias y responsabilidades cartográficas en España. Como se organiza la cartografía oficial en España, CICUM, Jaén.

- Merino, J.A., 2008. Province Map 1:200.000 with Geomedia Map Publisher, EuroCartoSIG, Budapest, Hungría.

- Merino, J.A. y González.-Matesanz, J., 2009. New cartography production methodologies from Province Map 1:200.000, ICC2009, Santiago de Chile. 